\title{
POSSIBILITIES TO MEASURE EVALUATING QUANTITIES OF THE MEDIUM FLOWING IN THE LANCE OUTFLOWS OF BLOWING SYSTEMS DESIGNED FOR HIGH-TEMPERATURE REACTORS
}

\author{
Ladislav KOVÁŘ, Pavel NOVÁK, Tomáš HAPLA, Jaroslav MELECKÝ \\ VŠB-Technical University of Ostrava, Faculty of Mechanical Engineering, Ostrava, Czech Republic, EU, \\ ladislav.kovar@vsb.cz, pavel.novak.st1@vsb.cz,tomas.hapla@vsb.cz, jaroslav.melecky@vsb.cz.
}

https://doi.org/10.37904/metal.2021.4079

\begin{abstract}
When designing blowing systems for a high-temperature reactor, we can primarily use mathematical tools that allow us to determine the state quantities of the flowing medium using a calculation. In many cases, however, it is necessary to verify these calculated quantities, e. g. by using a model. On the other hand, there is also a frequent situation occurring, when information needs to be obtained on an existing blowing system or its parts. In both cases, we must determine the basic parameters and properties of the flowing medium. These basic parameters include, in particular, the pressure and temperature of the flowing medium, and we address them as the so-called evaluating quantities. Specially designed probes are used to measure these evaluating quantities of the flowing medium, which differ from each other, depending on which flow parameter is being measured by the given probe. The submitted paper lists selected types of probes that can be used to measure the evaluating quantities of the lance outflows in the blowing systems which are used to blow gas media into the workspace of the high-temperature reactors. The possibility to compare calculated results to the measured ones supplies the designers of the blowing systems with important information on the reliability of their mathematical calculations and, thus, they can refine and correct their calculations so that they correspond as closely as possible to the real state.
\end{abstract}

Keywords: Gas blowing, lance outflows, blowing systems, press probes, temperature probes

\section{INTRODUCTION}

From the previous work in the field of the aerodynamics of lance outflows of free-flowing media for an ideal gas, it is known that the quantitative distribution of the determining quantities (Mach number $\mathbf{M}$, gas speed $\mathbf{v}$, temperature $\mathbf{T}$ etc.) is a function of the evaluating quantities (static pressure $\mathbf{p}$, stagnation pressure $p_{o}$ and stagnation temperature $T_{0}$ ), the distribution of which can be determined experimentally. Measurement of values $p_{0}, \mathbf{p}$ and $T_{0}$ for free outflow gas is the input for calculating the distribution of the determining quantities $\left(v, M, T, \rho v^{2}\right)$. The measurement method is based on the use of specially designed probes (sensors) applicable to subsonic and supersonic flowing medium, with which we can measure the relevant quantities [1-4]. The implementation of experimental measurement of evaluating quantities is a comprehensive and demanding task. This is particularly due to the installation of the measuring device and ensuring the necessary source (flow) of gas in case we want to take measurements directly on the work itself. For precise spatial determination of the position for the individual measured points or cross-section, we can use, for example, the results of mathematical calculations, or the results of one of the methods used for the visibility of the outflows [5].

\section{PRESSURE MEASUREMENT}

Relationship between stagnation pressure $\mathbf{p}_{0}$ and static pressure $\mathbf{p}$ is determined by the following formula, applicable for the so-called ideal gas 
$\frac{p_{0}}{p}=\left(1+\frac{k-1}{2} \cdot M^{2}\right)^{\frac{k}{k-1}}$

where:

$\mathrm{p}_{0}$ - stagnation pressure $(\mathrm{Pa})$,

$\mathrm{p}$ - static pressure $(\mathrm{Pa})$,

$M$ - Mach number (1),

$k$ - the ratio of specific heats at constant pressure and constant volume (1).

After modifying it, we get a mathematical expression in which the flow speed $\mathbf{v}$ occurs

$\frac{p_{0}}{p}=\left(1+\frac{(k-1) \cdot v^{2}}{2 k R T}\right)^{\frac{k}{k-1}}$

where:

$\mathrm{T}$ - static temperature $(\mathrm{K})$,

$R$ - gas constant, ratio between the universal gas constant and the molecular weight of the gas (1),

$v$ - flow speed $\left(m \cdot s^{-1}\right)$.

This means that if we know the stagnation pressure and the static pressure, then we can calculate the flow speed at the defined point using the following relationship

$v=\sqrt{\left[\left(\frac{p_{0}}{p}\right)^{\frac{k-1}{k}}-1\right] \cdot \frac{2 k R T}{k-1}}$

To measure the distribution of stagnation pressure $p_{0}$ on models and real works, probes on the principle of a Pitot tube are. These probes, in their shape, allow measurements in supersonic and subsonic areas of free outflow media. The dimensions of these probes are minimized to affect the gas flow as little as possible and, thus, to cause errors in the measurement. Miniature dimensions guarantee accurate sensing of the measures quantity at a given point of the flow cross-section. For example, the internal diameter $\emptyset \mathrm{D}$ is in the order of onetenth of a millimetre.

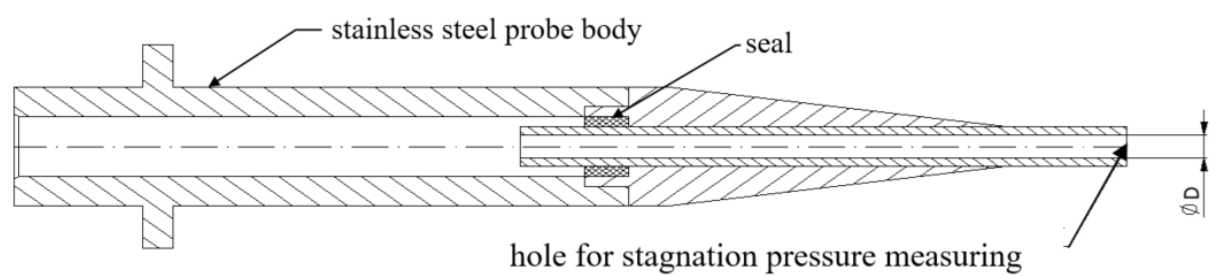

Figure 1 - Probe diagram for measuring the stagnation pressure

For their minimized dimensions, these probes are also suitable for measurements on models. A probe diagram for measuring the stagnation pressure is displayed in Figure 1. The stagnation pressure can be taken from the measuring probe to the converter $p_{o}-U$, where this pressure is transferred to electrical voltage using a pressure sensor. Thus, the obtained values can be recorded and further processed. A schematic example of a probe for measuring the distribution of static pressure $\mathbf{p}$, both in the free outflow axis and in the crosssections, is displayed in Figure 2. 


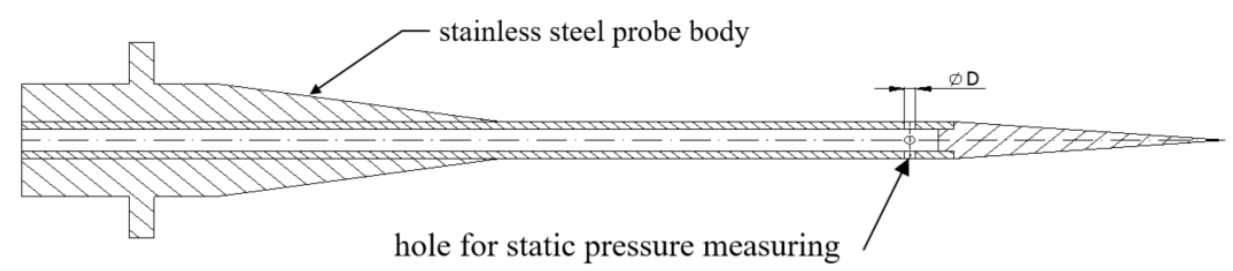

Figure 2 - Probe diagram for measuring static pressure

The probe is designed to measure the value of static pressure in both supersonic and subsonic free flow areas. The outer diameter of the probe active part is usually about one millimetre. The pressure is scanned via several holes with a diameter of 0.2 to $0.3 \mathrm{~mm}$. Technical conditions for measurement and data processing is analogous to probes for measuring stagnation pressure. In practice, probes are also used to measure stagnation and static pressure simultaneously. An example of such a probe is the Prandtl tube [6], [7]. For stagnation and static pressure probes to measure actual flow parameters, their longitudinal axis must be oriented precisely in the direction of the streamlines of the given flow, or flow speed vector [1],[4]. To determine the direction of the flow speed vector, multi-hole probes can be used. The principle of measurement is schematically illustrated in Figure 3. If the flow speed vector (streamlines axis) with the probe axis is at an angle $\boldsymbol{\alpha}$, then the pressure at points $A$ and $B$ will be different. By rotating the axis of the probe, we can achieve equalities between the two pressures. If the equality of the two pressures is achieved simultaneously and in a plane perpendicular to the cross-section plane shown in Figure 3, then the axis of the probe is oriented in the direction of the measured flow streamline (flow speed vector).

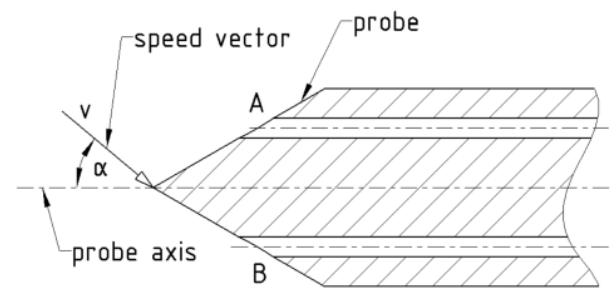

Figure 3 - Speed vector direction measurement scheme

\section{TEMPERATURE MEASUREMENT}

To measure the stagnation temperature $T_{0}$, there are many probe designs. An example of this is the probe diagram shown in Figure 4. A temperature sensor is located in the flow medium stagnation area. The temperature waveform can be recorded continuously at the appropriate speed of the probe feed. Stagnation temperature, static temperature and the speed expressed using the Mach number is to follow the relationship:

$\frac{T_{0}}{T}=\left(1+\frac{k-1}{2} \cdot M^{2}\right)$

Where:

$$
T_{0} \text { - stagnation temperature }(K) \text {, }
$$

Due to imperfect stagnation of the flow and heat exchange with the external environment, the probe measures the temperature $T_{m}$, the value of which lies between the temperatures $T_{0}$ and a real value of temperature $T$. Relationship between temperatures $T_{0}$ and $T$ is given by the formula:

$$
T_{0}=T+r_{k} \cdot \frac{v^{2}}{2 \cdot c_{p}}
$$


Where:
$r$ - restitution factor (1),
$\mathrm{C}_{\mathrm{p}}$ - specific heat capacity at constant pressure $\left(\mathrm{Jkg}^{-1} \mathrm{~K}^{-1}\right)$

The restitution factor value must be as close as possible to 1 for the widest possible range of Mach number $M$. If $r=1$, the relationship between $T_{0}$ and $T$ temperatures is given by:

$$
T_{0}=T+\frac{v^{2}}{2 \cdot c_{p}}
$$

In practice, combined probes are also used to measure the stagnation pressure and temperature at the same time.

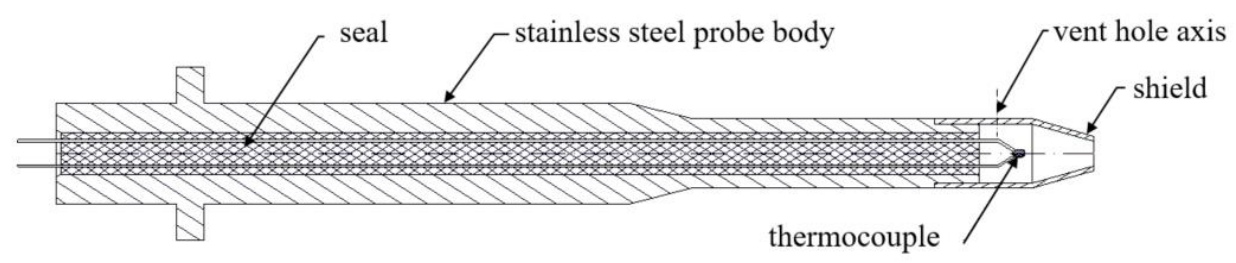

Figure 4 - Probe diagram for measuring the stagnation temperature

\section{SELECTED RESULTS OF MEASUREMENTS FOR EVALUATED QUANTITIES IN OUTFLOW GAS}

Figure 5 shows a diagram of the alternative arrangement of the measuring equipment used to measure evaluating quantities in gas flow streaming from individual refining nozzles. This whole measuring system can be divided into subsets of pneumatic, kinematic, and electrical.

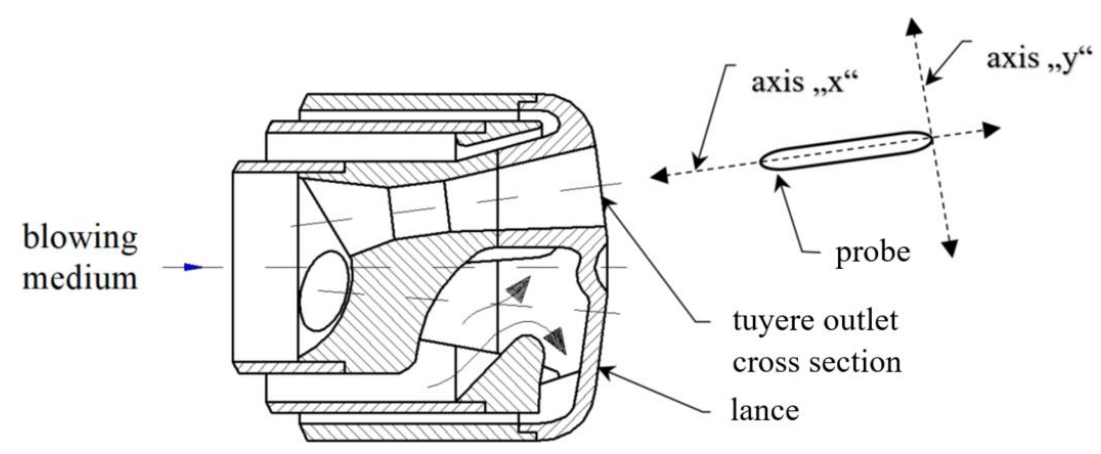

Figure 5 - Diagram of measuring probe movement before the nozzle

Pneumatic subset. It is used to produce, distribute, and regulate gas pressure to a constant value before a nozzle p_oL=const. In case we use air as a blown medium, it can be removed from an accumulator refilled with a compressor with sufficient power. Desired pressure value p_oL before the nozzle can be set with the help of a reducing valve. For measuring the flow rate of the blown gas, it is possible to use, for example, an orifice meter placed before the nozzle.

Kinematic subset. It is used to ensure even movement of the measuring probe in space. Automatic feed in all three directions of axes $\mathbf{x}, \mathbf{y}$ and $\mathbf{z}$ are used. Axis $\mathbf{x}$ is identical to the longitudinal line of the outflow $\mathbf{0}$. Axis $\mathbf{y}$ is perpendicular to the longitudinal line of the outlet flow $\mathbf{0}$, with the axes $\mathbf{x}$ and $\mathbf{y}$ form a horizontal plane. Axis $\mathbf{z}$ is perpendicular to the plane $x y$. The beginning of the coordinate system is on the longitudinal axis of the outflow $\mathbf{o}$. The nozzle head must be firmly fixed to the clamping device of the measuring instrument. 

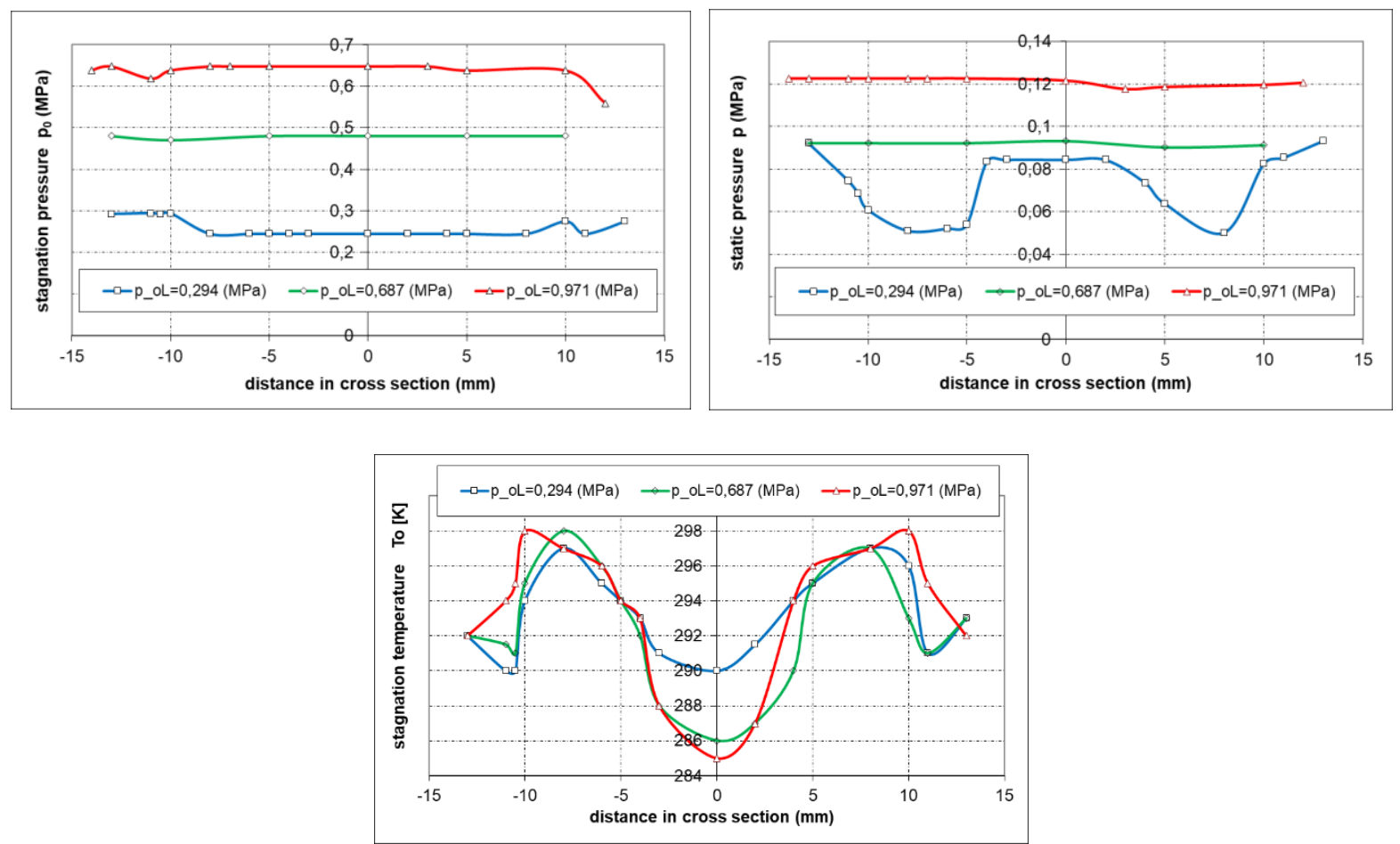

Figure 6 - Changes to the selected evaluating quantities in the nozzle output cross-section $x=0$

Electric subset. It is used to measure input and output evaluating quantities of gas, sensor positions, and values of pressures and ambient temperatures. To measure pressure and move, a pressure gauge fitted with resistance transmitters can be used. The evaluating quantities are usually recorded as a function of displacement.
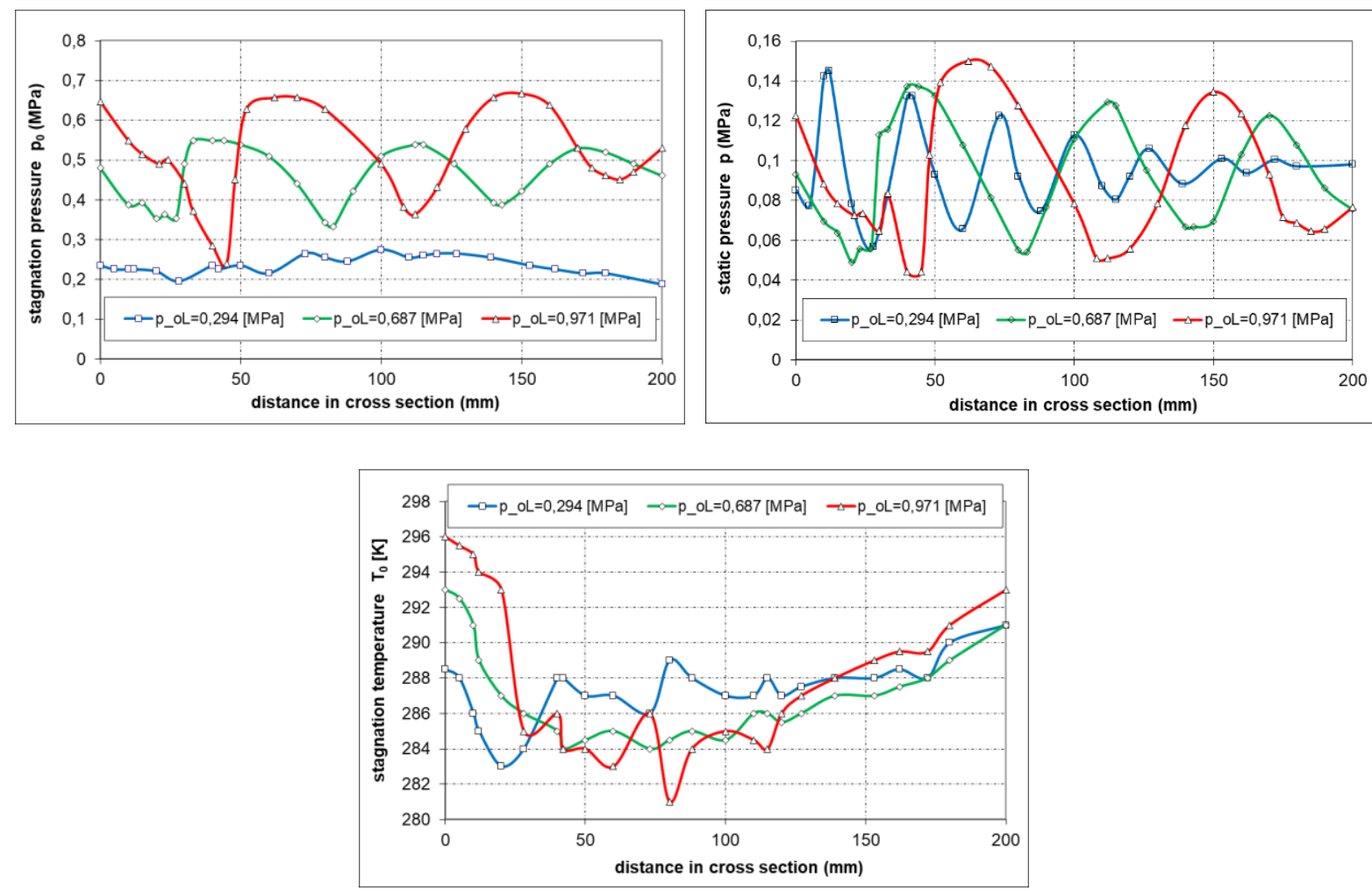

Figure 7 - Changes in the selected evaluating quantities of the outflow in different distances from the outlet cross-section in the direction of the longitudinal axis of the outflow o up to $200 \mathrm{~mm}$ 
Examples of selected courses of measured evaluating quantities, i.e., the stagnation pressure $\mathrm{p}_{0}$, static pressure $\mathbf{p}$ and stagnation temperature $T_{0}$, for selected media pressure values before the nozzle $p_{-}$oL, are displayed in Figure 6 and Figure 7 [8]. These evaluating quantities were measured both in the axis of the outflow and in selected cross-sections perpendicular to the longitudinal streamline axis of the outflow equipped with a measuring probe oriented at a given point in such a way that its longitudinal axis is in the direction of the flow speed vector. By calculation, you can determine the values of the $\mathrm{M}, \mathrm{v}$ and $\rho \mathrm{v}^{2}$.

\section{CONCLUSION}

To design the optimal dimensions of the blower nozzles, it may be necessary to complement the calculations and results by measuring on a model or on the work itself, in different nozzle working modes. Depending on the nature of distribution for the different types of evaluating quantities and the relevant determining quantities calculated from them, the e. g. the dynamic effect of the flow on the bath can be determined, change in the concentration of blown oxygen, and the most suitable nozzle position with an increase of its service life with reduced wear of the lining in the given thermal unit. The possibility and means of measuring evaluating quantities of the outflow jets allow this feedback to be realized to obtain valuable information about the real behaviour of a particular lance or nozzle design. In addition, these results help to improve the design of these special probes for pressure and temperature measurement, especially with regard to increasing their accuracy and reliability. The possibility of verifying the real parameters of outflow jets from lances and nozzles gives designers valuable feedback based on which it is possible to correct future designs of individual components for the gas flow systems.

\section{ACKNOWLEDGEMENTS}

\section{This paper was created with the support of the SGS project at the Department of Machine and Industrial Design, Faculty of Mechanical Engineering VSB-TUO.}

\section{REFERENCES}

[1] DEJČ, M.E. Technical gas dynamics. SNTL Praha, 1967, 660p.

[2] Measured Temperature - an overview. ScienceDirect Topics. ScienceDirect.com / Science, health and medical journals, full text articles and books. [online]. [viewed 2021-03-11]. Dostupné z: https://www.sciencedirect.com/topics/engineering/measured-temperature

[3] RUSSO, Giuseppe P. Aerodynamic measurements: from physical principles to turnkey instrumentation. Cambridge: Woodhead, 2011. Woodhead publishing in mechanical engineering. ISBN 978-1-84569-992-5.

[4] ASCHENBRUCK J. - HAUPTMANN T. SEUME - J.R. Influence of a multi-hole pressure probe on the flow field in axial-turbines, [online]. [viewed 2021-03-11]. Available from: https://www.euroturbo.eu/paper/ETC2015-155.pdf

[5] KOVÁŘ, Ladislav, NOVÁK, Pavel, HAPLA, Tomáš, FRIES, Jiří. Possibilities of analyzing the structure of lance outflows from the lances. In: METAL 2020. 29th. International Conference on Metallurgy and Materials. Brno: Czech Republic: TANGER Ltd., 2020, pp. 138-143.

[6] Př́rodovědecká fakulta JU. Mechanika tekutin. [online]. [viewed 2021-03-11]. Available from: https://www.prf.jcu.cz/data/files/18/441/4100mechanika9.pdf

[7] How does a Prandtl tube work? [online]. [viewed 2021-03-11]. Available from: https://www.tecscience.com/mechanics/gases-and-liquids/prandtl-tube/

[8] MIKOLAJEK, J. et al. Měření výtokových parametrů nové trysky T-pecí NHKG. Výzkumná zpráva. Ostrava. Fakulta strojní VŠB-TU Ostrava. 1978. 63s. 